\title{
ON GROUPS OF FINITE WEIGHT
}

\author{
P. KUTZKO
}

\begin{abstract}
A subset $S$ of a group $G$ is said to normally generate $G$ if the smallest normal subgroup of $G$ which contains $S$ is $G$ itself. If $\alpha$ is minimal with the property that there exist a set of cardinality $\alpha$ which normally generates $G$ then $G$ is said to have weight $\alpha$. It is shown that if $G$ is a group of finite weight and if the lattice of those normal subgroups of $G$ which are contained in the commutator subgroup $G^{\prime}$ of $G$ satisfies the minimum condition then the weight of $G$ is equal to the weight of $G / G^{\prime}$.
\end{abstract}

Let $G$ be a group and let $S$ be a subset of $G$. Then the normal closure, $\langle S\rangle$, of $S$ in $G$ is defined to be the smallest normal subgroup of $G$ which contains $S$. If $G$ is a nontrivial group and if the cardinal $\alpha$ is minimal with the property that $G$ contain a subset of cardinality $\alpha$ whose normal closure is $G$, then following [1] we say that $G$ has weight $\alpha$ and write $w(G)=\alpha$; we define the weight of the trivial group to be one. Questions concerning the weight of a group and especially the relation between the weight of a group and that of its abelianization arise naturally in the study of knot groups [1], [3]. In particular, it is conjectured by González-Acuña in [1] that for every finite group $G$, $w(G)=1$ if and only if $w\left(G / G^{\prime}\right)=\mathrm{i}$. (An example of Kervaire [2] shows that the analogous conjecture for finitely generated, infinite groups is false.) The purpose of this paper is to prove the following more general result.

THEOREM. Let $G$ be a group of finite weight and let $\mathcal{L}$ be the lattice of normal subgroups of $G$ which are contained in the commutator subgroup $G^{\prime}$ of $G$. Then if $\mathcal{L}$ satisfies the minimum condition, $w(G)=w\left(G / G^{\prime}\right)$.

Proof. Let $N$ be a normal subgroup of $G$ which is contained in $G^{\prime}$ and is minimal with the property that $w(G / N)=w\left(G / G^{\prime}\right)$. We wish to show that $N=(1)$. Set $w=w\left(G / G^{\prime}\right)$ and let $\bar{S}=\left\{\bar{g}_{1}, \ldots, \bar{g}_{w}\right\}$ be a subset of $G / N$ such that $\langle\bar{S}\rangle=G / N$. For $i=1,2, \ldots, w$, pick $g_{i}$ in $G$ which maps onto $\bar{g}_{i}$ under the natural map from $G$ to $G / N$, set $S=\left\{g_{1}, \ldots, g_{w}\right\}$ and let $K=\langle S\rangle \cap N$. Then since $G=N\langle S\rangle$, if $K=N$, then $G=\langle S\rangle$ so that $N=$ (1). Suppose now that $K \neq N$. Then since $N / K$ is isomorphic to $G /\langle S\rangle, N / K$ has finite weight from which we may conclude that $N$ has a proper normal subgroup $L$ which contains $K$ such that $w(N / L)=1$. We note that $L$ is in fact a normal subgroup of $G$ since $G / K$ is the direct product of its subgroups $N / K$ and $\langle S\rangle / K$. We now show that $w(G / L)=w(G / N)$ from which our theorem will follow immediately.

First, we note that $G / L$ is naturally the direct product of its subgroups

Received by the editors May 2, 1975.

AMS (MOS) subject classifications (1970). Primary 20F05; Secondary 20F15. 
$\langle S\rangle / K$ and $N / L$ and that $N / L$ is contained in $(G / L)^{\prime}$. From this it follows that $N / L$ is contained in $(N / L)^{\prime}$ so that $N / L$ is perfect. Pick $x$ in $N$ so that $N / L$ is the normal closure of the image $\bar{x}$ in $N / L$ of $x$ under the natural map from $N$ to $N / L$; let $\bar{g}_{1}$ be the image of $g_{1}$ in $\langle S\rangle / K$ under the natural map from $\langle S\rangle$ to $\langle S\rangle / K$ and let $\bar{y}$ be any element of $N / L$. Then $\left[\bar{x} \bar{g}_{1}, \bar{y}\right]=[\bar{x}, \bar{y}]$ in $G / L$, and thus if we denote the normal closure in $N / L$ of the set of elements $[\bar{x}, \bar{y}], \bar{y}$ in $N / L$, by $\bar{M}$, then we see that $\bar{M}$ is contained in $\left\langle\bar{x} \bar{g}_{1}\right\rangle$. However, since $N / L=\langle\bar{x}\rangle,(N / L) / \bar{M}$ is cyclic and since $N / L$ is perfect, we conclude that $N / L=\bar{M}$ so that $N / L$ is contained in $\left\langle\bar{x} \bar{g}_{1}\right\rangle$ and, hence, $N$ is contained in $\left\langle x g_{1}\right\rangle L$.

Setting $T=\left\langle x g_{1}, g_{2}, \ldots, g_{w}\right\rangle$ and noting that $x$ is an element of $N$ we see that $G=\langle S\rangle N=\langle T\rangle N=\langle T\rangle L$. Thus $G / L$ is the normal closure of the image of $T$ in $G / L$ under the natural map from $G$ to $G / L$ and since $w(G / L) \geqslant w(G / N)$, we have shown that $w(G / L)=w(G / N)=w\left(G / G^{\prime}\right)$.

\section{REFERENCES}

1. F. González-Acuña, Homomorphs of knot groups (to appear).

2. M. A. Kervaire, On higher dimensional knots, Differential and Combinatorial Topology (A Sympos. in Honor of Marston Morse), Princeton Univ. Press, Princeton, N.J., 1965, pp. 105-109. MR 31 \# 2732.

3. L. Neuwirth, Knot groups, Ann. of Math. Studies, no. 56, Princeton Univ. Press, Princeton, N.J., 1965. MR 31 \# 734.

Division of Mathematical Sciences, University of Iowa, Iowa City, Iowa 52242 